Bundesgesundheitsbl 2011 · 54:1269-1269

DOI 10.1007/s00103-011-1367-1

Online publiziert: 23. November 2011

(c) Springer-Verlag 2011

\section{R. von Kries}

Institut für Soziale Pädiatrie und Jugendmedizin, Abteilung für Epidemiologie des Kindes- und Jugendalters, Ludwig-Maximilians-Universität München, München

\title{
Schlaf und Gesundheit
}

\section{Liebe Leserin, lieber Leser,}

Schlaf und Schlafstörungen sind in der modernen Gesellschaft mit ihren erheblichen adaptiven Anforderungen unter anderem durch das Berufsleben und durch Lärmbelastung in der Umwelt ein Thema von allgemeinem Interesse. Für die medizinische Profession wurde Schlaf zum Thema, da medizinische Ursachen nicht nur zu Schlafstörungen führen können, sondern Schlafstörungen beziehungsweise Variabilitäten im Schlafverhalten andererseits auch gesundheitliche Effekte haben können.

Neue wissenschaftliche Erkenntnisse haben unser Verständnis der Physiologie des Schlafs verbessert. Dies ist das Thema des Beitrags von PD Dr. Rodenbeck. Die Mechanismen des Einschlafens und Aufwachens sowie die hormonellen Veränderungen im Schlaf wurden intensiv untersucht. Die Bedeutung unterschiedlicher Schlafphasen für die Verarbeitung von Erlebtem, insbesondere für Gedächtnisleistungen, und deren Regulation wird auch im Kontext ihrer Relevanz für die Therapie dargestellt. Die Beurteilung von Schlafdauer und Qualität des Schlafes ist nicht trivial. Dr. Heitmann und Kollegen geben eine Übersicht über die subjektiven und objektiven Methoden der Schlafbeurteilung und deren Relevanz für epidemiologische, wissenschaftliche und klinische Fragestellungen. Eine Übersicht über die häufigsten schlafmedizinischen Erkrankungen gibt der Beitrag von Prof. Mayer. Häufigkeit, Symptome, Pathophysiologie und Therapie von Restless-Legs-Syndrom, Insomnien und Schlafapnoen werden dargestellt.

Kurze und offenbar auch lange Schlafdauer ist mit einem erhöhten kardiovaskulären Risiko assoziiert, wie in dem Beitrag von Prof. Riemann und Kollegen aus-

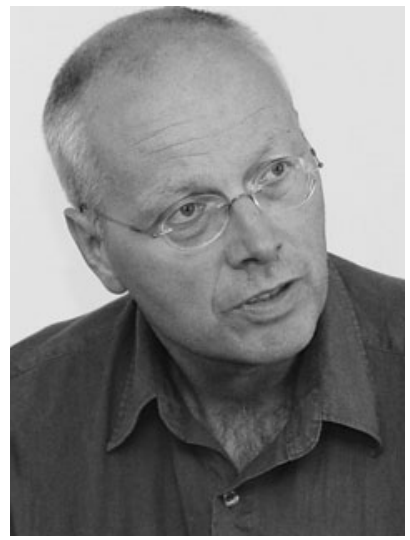

geführt wird. Interessant sind auch die neuen Daten zur Assoziation von Depression und Insomnie: Depressive Störungen können zu Schlafstörungen führen; offenbar erhöhen aber auch Schlafstörungen das Risiko für depressive Symptome.

Frau Gottschalk und Kollegen geben eine Übersicht über Schlafstörungen im Kindesalter, wobei neben den Störungen, die auch bei Erwachsenen auftreten, spezifische Probleme im Kindesalter wie die altersspezifische Apnoeproblematik bei Frühgeborenen, angeborene Hypoventilationssyndrome und Parasomnien hervorzuheben sind. Besonders häufig sind Schlafstörungen im Alter. Frau Prof. Happe erläutert die häufigsten Ursachen, die notwendige Diagnostik und therapeutische Optionen. Darüber hinaus werden ausgewählte spezifische Schlafstörungen im Alter dargestellt. Exogene Ursachen von Schlafstörungen werden in den folgenden zwei Beiträgen dargestellt. Frau Kohlhuber und PD Dr. Bolte stellen die epidemiologischen, pathophysiologischen und empirischen Kenntnisse zu Lärmbelastung und Schlafstörungen dar. Prof. Riemann und Dr. Nissen geben eine Übersicht zu substanzinduzierten Schlafstörungen und Schlafmittelmissbrauch. Eine herausgehobene Darstellung wurde für das Restless-Legs-Syndrom und die Zusammenhänge von Schlaf und Übergewicht gewählt. Dr. Remi stellt neben eine Übersicht zu den epidemiologischen, diagnostischen und therapeutischen Aspekten eine Darstellung der versorgungs- und gesundheitsökonomischen Bedeutung dieser Erkrankung, die von besonderer PublicHealth-Relevanz ist. Im Kontext der Adipositasepidemie steht die Darstellung der Zusammenhänge von Schlafmangel und Adipositasrisiko. Die empirische und experimentelle Evidenz für diese Zusammenhänge und welche potenziellen Implikationen sich hieraus für die Prävention und Therapie ergeben könnten, stellen Frau Hense und Dr. Bayer dar.

Die Beiträge verdeutlichen die eminente gesundheitliche Relevanz von Schlaf und Schlafstörungen. Wird das Gesundheitssystem in Deutschland diesen Herausforderungen gerecht - wo sind Verbesserung möglich und notwendig? Diese Thematik greift der letzte Beitrag von Prof. Fitze und Prof. Penzel auf: Vieles ist erreicht, manches noch zu tun.

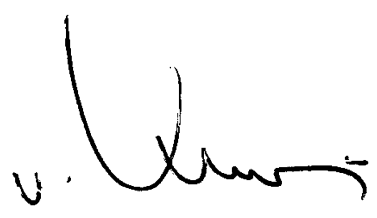

Ihr Rüdiger von Kries

\section{Korrespondenzadresse \\ Prof. Dr. R. von Kries}

Institut für Soziale Pädiatrie und Jugendmedizin, Abteilung für Epidemiologie des Kindes- und Jugendalters, Ludwig-Maximilians-Universität München Heiglhofstr. 63, 81377 München

ruediger.kries@med.uni-muenchen.de 